Published in final edited form as:

J Surg Res. 2019 April ; 236: 332-339. doi:10.1016/j.jss.2018.11.048.

\title{
EVIDENCE VS. PRACTICE IN EARLY DRAIN REMOVAL FOLLOWING PANCREATECTOMY
}

\author{
N Villafane-Ferriol ${ }^{\mathrm{a}}$, KA Baugh ${ }^{\mathrm{a}}$, AL McElhany $^{\mathrm{a}}$, G Van Buren II ${ }^{\mathrm{a}}$, A Fang $^{\mathrm{a}}$, EK Tashakori ${ }^{\mathrm{a}}$, \\ JE Mendez Reyes ${ }^{\mathrm{a}}$, HS Tran Cao ${ }^{\mathrm{a}}$, EJ Silberfein ${ }^{\mathrm{a}}$, N Massarweh ${ }^{\mathrm{a}}, \mathrm{C} \mathrm{Hsu}^{\mathrm{a}}$, O Barakat ${ }^{\mathrm{a}}, \mathrm{C}$ \\ Schmidt $^{\mathrm{b}}$, NJ Zyromski ${ }^{\mathrm{c}}$, M Dillhoff $^{\mathrm{b}}$, JA Villarreal ${ }^{\mathrm{a}}$, and WE Fisher ${ }^{\mathrm{a}}$ \\ a.Baylor College of Medicine Michael E. DeBakey Department of Surgery, One Baylor Plaza Suite \\ 404D, Houston, TX 77030 \\ b. Ohio State University Department of Surgery, M256 Starling Loving Hall, 320W $10^{\text {th }}$ Avenue, \\ Columbus, Ohio 43210 \\ c. Indiana University School of Medicine Department of Surgery, 545 Barhill Drive EH 519, \\ Indianapolis, IN 46202
}

\begin{abstract}
Background: Early drain removal when postoperative day (POD) 1 drain fluid amylase (DFA) was $\leq 5000 \mathrm{U} / \mathrm{L}$ reduced complications in a previous randomized controlled trial. We hypothesized that most surgeons continue to remove drains late and this is associated with inferior outcomes.

Methods: We assessed the practice of surgeons in a prospectively maintained pancreas surgery registry to determine the association between timing of drain removal with demographics, comorbidities, and complications. We selected patients with POD1 DFA $\leq 5000 \mathrm{U} / \mathrm{L}$ and excluded those without drains, and subjects without data on POD1 DFA or timing of drain removal. Early drain removal was defined as $\leq$ POD5.
\end{abstract}

Results: 244 patients met inclusion criteria. Only 90 (37\%) had drains removed early. Estimated blood loss was greater in the late removal group $(190 \mathrm{~mL}$ vs $100 \mathrm{~mL}, \mathrm{p}=0.005)$ and pathological

Corresponding Author and Reprints: William E. Fisher, M.D., F.A.C.S, Professor and Chief, Division of General Surgery, George L. Jordan, M.D., Chair of General Surgery, Director, Elkins Pancreas Center, Vice Chair for Clinical Affairs, Michael E. DeBakey Department of Surgery, Baylor College of Medicine, 6620 Main Street, Suite 1425, Houston, Texas 77030, wfisher@ bcm.edu. Contributions:

Nicole Villafane-Ferriol (NVF), Katherine A. Baugh (KAB), Amy L. McElhany (ALM), George Van Buren, II (GVB), Andrew Fang (AF), Erisha K. Tashakori (EKT), Jose E. Mendez-Reyes (JEMR), Hop S. Tran Cao (HSTC), Eric J. Silberfein (EJS), Nader Massarweh (NM), Cary Hsu (CH), Omar Barakat (OB), Carl Schmidt (CS), Nicholas J. Zyromski (NJZ), Mary Dillhoff (MD), Joshua A. Villarreal (JAV), William E. Fisher (WEF)

Concept or design of work: NVF, ALM, GVB, WEF

Acquisition, analysis, or interpretation of work: NVF, KAB, ALM, GVB, AF, EKT, JEMR, HSTC, EJS, NM, CH, OB, CS, NJZ, MD, JAV, WEF Drafting of manuscript: NVF, AM, GVB, JEMR, WEF

Revision of manuscript: NVF, KAB, ALM, GVB, AF, EKT, JEMR, HSTC, EJS, NM, CH, OB, CS, NJZ, MD, JAV, WEF

Final approval: NVF, KAB, ALM, GVB, AF, EKT, JEMR, HSTC, EJS, NM, CH, OB, CS, NJZ, MD, JAV, WEF

Disclosures:

The authors have no conflict of interest related to the work described in this article.

Publisher's Disclaimer: This is a PDF file of an unedited manuscript that has been accepted for publication. As a service to our customers we are providing this early version of the manuscript. The manuscript will undergo copyediting, typesetting, and review of the resulting proof before it is published in its final citable form. Please note that during the production process errors may be discovered which could affect the content, and all legal disclaimers that apply to the journal pertain. 
findings associated with soft gland texture were more frequent (97(63\%) vs 35(39\%), p < 0.0001). Patients in the late drain removal group had more complications $(84(55 \%)$ vs $30(33 \%), \mathrm{p}=0.001)$ including pancreatic fistula (55(36\%) vs 4(4\%), $\mathrm{p}<0.0001)$, delayed gastric emptying (27(18\%) vs $3(3 \%), p=0.002$ ), and longer length of stay ( 7 days vs 5 days, $\mathrm{p}<0.0001)$. In subset analysis for procedure type, complications and pancreatic fistula remained significant for both pancreatoduodenectomy and distal pancreatectomy.

Conclusion: Despite level 1 data suggesting improved outcomes with early removal when POD1 DFA is $\leq 5000 \mathrm{U} / \mathrm{L}$, experienced pancreas surgeons more frequently removed drains late. This practice was associated with known risk factors (EBL, soft pancreas) and may be associated with inferior outcomes suggesting potential for improvement.

\section{Introduction:}

Within complex pancreatic surgery, the most significant complication is a post-operative pancreatic fistula (POPF). ${ }^{1-3}$ Many modifications have been proposed throughout the years in an attempt to prevent POPF including variations in surgical technique. However, fistulas still occur in $10 \%$ of patients and cause significant morbidity. ${ }^{4}$ Historically, the use of abdominal drains mitigated that morbidity but a growing appreciation as to the unnecessary or detrimental nature of drains in other operations including splenectomy and gastrectomy called into question the use of drains in complex pancreatic surgery. ${ }^{4-5}$

Recently, concerns that drains may increase post-operative complications rather than mitigate them caused a decline in the usage of prophylactic abdominal drains. A poll of experienced pancreatic surgeons revealed that $27 \%$ elected for selective use of abdominal drains with $51 \%$ removing them earlier than post-operative day 3 (POD3). ${ }^{6}$ Support for this practice stemmed from the idea that drains provide not only a portal of entry for bacteria but also generate considerable negative pressure potentially causing fistula formation. However, post-operative day (POD) 1 drain fluid amylase (DFA) concentration greater than $5000 \mathrm{U} / \mathrm{L}$ has been associated with an increased risk of clinically relevant postoperative pancreatic fistula (CR-POPF) and in those patients, the elimination of prophylactic drains would expose them to considerable risk. ${ }^{7,8}$ Therefore, the selective use of intra-operative drains with early drain removal in patients with negligible fistula risk provides a potential solution.

However, the current literature evaluating outcomes in pancreatectomy patients after early drain removal is limited. Indeed, early drain removal in selected patients has been associated with reduced postoperative complications after pancreatectomy. ${ }^{9}$ Only one randomized prospective trial by Bassi and colleagues has assessed the safety of early drain removal. ${ }^{9}$ Despite results showing improved outcomes, many surgeons remain reluctant to remove intraperitoneal drains early in the postoperative period. We hypothesized that in low risk patients (POD1 DFA $\leq 5000 \mathrm{U} / \mathrm{L}$ ), late drain removal (> POD 5) would be associated with worse outcomes including an increased rate of CR-POPF and intra-abdominal abscess.

\section{Methods:}

We queried data from a prospectively maintained pancreas surgery registry at a high-volume academic pancreas center, Baylor College of Medicine, from January 2006 to December 
2016. The electronic web-based database contains data on patient demographics, clinical history, past medical history, family and social history, physical exam findings, diagnostic tests and imaging, as well as detailed data on operative interventions and pathologic data. All complications within 60 days of surgery are prospectively recorded and are graded using the Accordion Severity Grading for Surgical Complications ${ }^{10}$ and International Study Group on Pancreatic Fistula (ISGPF)/International Study Group of Pancreatic Surgery (ISGPS) definitions for pancreatic fistula and delayed gastric emptying. ${ }^{11,12}$ Survival is recorded up to 90 days for all patients and until death for patients with cancer. After obtaining informed consent, data is entered into the database in real time by trained data analysts under the supervision of the surgeons. All data are backed up by source documents and the accuracy of data entered to the electronic database is periodically reviewed. ${ }^{13}$ Institutional Review Boards at our institution granted permission to conduct this study (IRB H-38662).

In the current study, we included patients who underwent pancreatoduodenectomy or distal pancreatectomy, for benign and malignant disease, who had a POD1 DFA $\leq 5000 \mathrm{U} / \mathrm{L}$. We excluded patients without intraperitoneal drains and those without available data on timing of drain removal. Baseline demographics and past medical history were obtained from the database. Perioperative characteristics included pancreatic texture, duct size, pathologic diagnosis, EBL, intra-operative transfusion requirement, American Society of Anesthesiologists (ASA) score, and procedure length. Complications were assessed at 60 days. Chi square or Fisher's exact test, when appropriate, was used to analyze categorical variables. For continuous variables, the student's t-test or Mann-Whitney test were used to evaluate continuous variables. A p-value of $<0.05$ was considered statistically significant. All statistical analyses were performed using SPSS v24 (IBM Corp. Armonk NY, USA).

\section{Results:}

We identified 687 patients in our database who underwent pancreatoduodenectomy or distal pancreatectomy. We excluded 189 patients who did not have intraperitoneal drains placed at the time of pancreatectomy, 146 without data on timing of drain removal, 62 without POD1 DFA value data, and 46 with POD1 DFA > $5000 \mathrm{U} / \mathrm{L}$. This resulted in 244 patients meeting inclusion criteria with a POD1 DFA $\leq 5000 \mathrm{U} / \mathrm{L}$ (Fig 1). Of these, 176 (72\%) underwent pancreatoduodenectomy and 68 (28\%) underwent distal pancreatectomy. Patients were divided into two groups based on timing of drain removal. Early removal was defined as on or before POD5 (90 (37\%)), and late removal was defined as after POD5 (154, (63\%)). Most drains in the early removal group were removed by POD 4 (POD 3-5 IQR) while in the late group, most were removed by POD 11 (POD 8-17 IQR). As the practice progressed, there was a trend toward earlier drain removal. Prior to $2010,6.5 \%$ of drains were removed early which increased to $46.2 \%$ after publication of the Bassi study. ${ }^{9}$ Additionally, surgeon experience and case volume did not affect timing of drain removal. Three surgeons were studied of which 1 is junior faculty with 5 years of experience performing an average of 20 complex pancreas surgeries a year, and 2 senior faculty both with 20 years of experience and an average of over 40 cases a year. The junior faculty removed the drain early in $44 \%$ of patients while the senior faculty removed the drain in $35 \%(\mathrm{P}=0.204)$. 
The overall median POD1 DFA was 328 U/L (58-1270 U/L IQR). Median POD1 DFA was $176 \mathrm{U} / \mathrm{L}$ (37-691 U/L IQR) in the early group and 599 U/L (116-1755 U/L IQR) in the late group ( $\mathrm{p}<0.0001$ ). POD3 DFA data was available for $98 \%$ of patients in the early drain removal group and $94 \%$ in the late removal group. In those with early drain removal, median POD3 DFA was 21.5 U/L (IQR 10-72.5) among pancreatoduodenectomies and $104 \mathrm{U} / \mathrm{L}$ (IQR 46-176) for distal pancreatectomies with only 4 (4\%) of patients with a DFA value three times the upper limit of normal. Among the late removal group, median POD3 DFA was $154 \mathrm{U} / \mathrm{L}$ (IQR 33.5-389.5) for pancreatoduodenectomies and $236 \mathrm{U} / \mathrm{L}$ (IQR 99.5-790) for distal pancreatectomies with 42(27\%) with an elevated DFA on POD3.

Table 1 shows the baseline characteristics of the study population. There was no significant difference in age, gender, ethnicity, race, or distribution of comorbidities between the early and late drain removal groups. Patients in the late removal group had greater EBL (190 vs $100 \mathrm{ml}, \mathrm{p}=0.005)$ and required intra-operative transfusions more frequently $(20(13 \%)$ vs 4(4\%), $\mathrm{p}=0.03)$. The difference in EBL persisted in subset analysis for pancreatoduodenectomy and distal pancreatectomy (Table 2). Patients in the late removal group were also more likely to have a soft pancreatic texture (92(60\%) vs 44(49\%), $\mathrm{p}=0.04$ ). This persisted in subset analysis for pancreatoduodenectomy patients but not for distal pancreatectomy. Pre-operative antibiotics were variable but Ertapenem and Meropenem predominated with Ertapenem used significantly more in the late drain removal group (62\% vs $77 \%, \mathrm{p}=0.012$ ) and Meropenem used in the early removal group (13(15\%) vs $2(1 \%)$, $\mathrm{p}<0.001)$. Intra-operative octreotide usage was more frequent in the late removal group (43(28\%) vs $8(9 \%), \mathrm{p}<0.001)$ in the overall cohort and in the pancreatoduodenectomy subset $(41(35 \%)$ vs $6(10 \%), \mathrm{p}<0.001$. However, operative technique was not statistically significant between the early and late removal groups in either pancreatoduodenectomy or distal pancreatectomy patients.

Overall morbidity (excluding Grade A pancreatic fistula) was higher in the late removal group for both pancreatoduodenectomy and distal pancreatectomy patients (Table 3). More patients in the late removal group had a pancreatic fistula of any grade (55(36\%) vs 4(4\%), $\mathrm{p}<0.0001$ ), but there was no difference in the rates of CR-POPF (ISGPF Grade B or C). In those with a CR-POPF, $60 \%$ (9 of 15) were diagnosed prior to POD7 all of which required late drain removal but only accounted for 5.8\% (9 of 150) of patients with late drain removal. Three patients underwent early drain removal but were diagnosed with a CR-POPF in the second to third post-operative week. Delayed gastric emptying was also more prevalent in the late removal group (27(18\%) vs $3(3 \%), \mathrm{p}=0.002)$. This difference persisted after subset analysis based on procedure type (Table 3). The difference in length of stay (LOS) between the two groups was also significant, with longer LOS in the late removal group ( 7 days vs 5 days; $p<0.0001$ ). Overall, there was no difference in rate of intraabdominal abscess formation. However, on subset analysis for subjects undergoing distal pancreatectomy, late drain removal had a higher incidence of intra-abdominal abscess $(5(14 \%)$ vs $0, \mathrm{p}=0.05)$ 


\section{Discussion:}

Early drain removal following pancreatectomy when POD1 DFA was $\leq 5000 \mathrm{U} / \mathrm{L}$ resulted in reduced complications in a previous randomized controlled trial. ${ }^{9}$ We hypothesized that despite these data, most surgeons continue to remove drains late and that this is associated with inferior outcomes. Our retrospective study showed that experienced pancreatic surgeons removed drains early only $37 \%$ of the time when the POD1 DFA was $\leq 5000 \mathrm{U} / \mathrm{L}$ despite surgeon experience and volume. This practice was associated with known risk factors for pancreatic fistula, such as increased EBL and soft gland texture, and may be associated with inferior outcomes suggesting potential for improvement.

Drains are placed following pancreatic resection because some surgeons believe they will control a postoperative leak of pancreatic secretions and decrease the incidence of postoperative peripancreatic fluid collections, abscesses, or erosion of retroperitoneal vessels resulting in postoperative hemorrhage. However, some surgeons have questioned this position and believe that drains, particularly if left in place for a prolonged period, can erode into adjacent structures and increase the incidence of postoperative pancreatic fistula (POPF) or serve as an avenue to introduce bacteria into a sterile pancreatic fluid collection, thus increasing the incidence of intra-abdominal abscesses.

Kawai et al. were the first to report improved outcomes with early drain removal after pancreatoduodenectomy. ${ }^{14}$ In this prospective cohort study, early drain removal was defined as removal on POD4 and late as on or after POD8, regardless of DFA value. The rates of POPF (3.6\% vs $23 \%, \mathrm{p}=0.004)$ and intra-abdominal abscess $(7.7 \%$ vs $38 \%, \mathrm{p}=0.003)$ were significantly lower in the early removal group. The authors also reported a $17 \%$ rate of CRPOPF in the late drain removal group compared to $2 \%$ in the early removal group.

A subsequent prospective cohort study by Adachi et al. focusing on subjects undergoing distal pancreatectomy also demonstrated improvement with early drain removal. ${ }^{15}$ The authors defined early drain removal on POD1 and late removal on $\geq$ POD5, early removal was once again favored with a $0 \%$ incidence of CR-POPF in the early group compared to $16 \%$ in the late removal group.

The study by Bassi and colleagues randomized patients undergoing either pancreatoduodenectomy or distal pancreatectomy into early and late removal groups on POD 3. ${ }^{9}$ In this study, which provides higher level of evidence, the authors found an association between early drain removal and decreased rates of pancreatic fistula (early $1.8 \%$ vs late $26 \%, \mathrm{p}=0.0001$; OR 20) and intra-abdominal complications (early $12.2 \%$ vs late $53 \%, \mathrm{p}=0.001$; OR 7.9).

McMillan and colleagues conducted a multicenter prospective study to evaluate a drain management strategy combining selective and early drain removal in pancreatoduodenectomy patients. ${ }^{16}$ They employed the Fistula Risk Score (FRS), which uses intraoperative characteristics including EBL, pancreatic duct size, gland texture, and pathology. ${ }^{17}$ The authors abandoned intraperitoneal drain placement in negligible-low risk patients and removed drains early (POD3) in those with moderate-high risk if their POD1 DFA was $\leq 5000 \mathrm{U} / \mathrm{L}$. The authors compared their study population to a retrospective cohort 
and found the CR-POPF rate to be lower after implementation of the drain management protocol.

Beane et al. recently performed a retrospective analysis of the American College of Surgeons-National Surgical Quality Improvement Program (ACS-NSQIP) database on outcomes for early versus delayed drain removal in pancreatoduodenectomy patients. ${ }^{18}$ Patients with POD 1 DFA $\leq 5000 \mathrm{U} / \mathrm{L}$ whose drains were removed by POD3 were propensity score matched with patients whose drains were removed after POD3. Early drain removal when POD 1 DFA was $\leq 5000 \mathrm{U} / \mathrm{L}$ was associated with reduced overall morbidity (35.3\% vs $52.3 \%, \mathrm{p}=0.01)$, length of stay ( 6 vs 8 days, $\mathrm{p}<0.01)$, and CR-POPF ( $0.9 \%$ vs $7.9 \%, \mathrm{p}=0.02) .{ }^{18}$ Despite the strengths of the study, important factors including surgeon experience and case volume were not included. A surgeon's practice evolves through experience and newer surgeons with lower case volumes may hesitate to remove a drain earlier due to the concern for complications. Using an institutional database, we were able to perform granular analysis and show that surgeon experience did not contribute to timing of removal.

These previous studies suggest that late drain removal in subjects at a lower risk for fistula may increase the occurrence of complications including fistula and intra-abdominal abscess. However, in the current study, surgeons removed drains late even in the subset of patients with POD1 DFA $\leq 5000 \mathrm{U} / \mathrm{L}$. The overall median POD1 DFA in our study suggests that, if POD1 DFA value was not very low, surgeons may have been concerned about early drain removal as evidenced by a greater percentage of patients with an elevated POD3 amylase levels in the late group (27\%) vs the early group (4\%). Additionally, patient specific factors such as EBL, soft pancreatic texture, or concerns regarding the pancreatic anastomosis or transection line may have influenced their clinical decision to leave drains in place longer despite a low POD1 DFA. These factors could not be thoroughly assessed in this retrospective study. Another potential bias, given the retrospective nature of this study, is evolution of practice, as time progressed surgeons elected to remove drains early more often. The higher morbidity in the late removal group supports the concept that prolonged use of drains could be detrimental in patients at low risk of pancreatic fistula based on POD1 DFA concentration. An alternative explanation is that, perhaps driven by other patient specific characteristics signifying increased POPF risk, surgeons made wise decisions to leave drains in place since these patients indeed more frequently went on to have complications. The retrospective nature of this study does not allow us to measure all factors that come together in aggregate to influence surgical decision making.

In this retrospective study, we demonstrated that experienced pancreas surgeons frequently choose to remove drains late when POD1 DFA is $\leq 5000 \mathrm{U} / \mathrm{L}$ despite level 1 data suggesting improved outcomes with early removal. This practice was associated with inferior outcomes suggesting potential for improvement. We conclude that early drain removal should be encouraged when POD1 DFA is $\leq 5000$ U/L. Data from additional multicenter randomized prospective trials may be needed to disseminate this change in surgical practice. 


\section{Acknowledgments}

This work was supported by the National Institute of Health (NIH) and the National Institute of Diabetes and Digestive and Kidney Diseases (NIDDK) (5 R21 DK106650-02). The content is solely the responsibility of the authors and does not necessarily represent the official views of the NIH or NIDDK.

\section{References:}

1. DeOliveira ML, Winter JM, Schafer M, et al. Assessment of complications after pancreatic surgery: a novel grading system applied to 633 patients undergoing pancreaticoduodenectomy. Ann Surg 2006;244(6):931-937. [PubMed: 17122618]

2. Lermite E, Sommacale D, Piardi T, et al. Complications after pancreatic resection: diagnosis, prevention and management. Clin Res Hepatol Gastroenterol 2013;37(3):230-239. [PubMed: 23415988]

3. Malleo G, Pulvirenti A, Marchegiani G, Butturini G, Salvia R, Bassi C. Diagnosis and management of postoperative pancreatic fistula. Langenbeck's Arch Surg 2014;399(7):801-810. [PubMed: 25173359]

4. Fisher WE. Intraperitoneal Drainage and Pancreatic Resection. Adv Surg 2018;52(1):205-222. [PubMed: 30098613]

5. Conlon KC, Labow D, Leung D, et al. Prospective randomized clinical trial of the value of intraperitoneal drainage after pancreatic resection. Ann Surg 2001;234:487-93. [PubMed: 11573042]

6. McMillan MT, Malleo G, Bassi C, et al. Defining the practice of pancreaticoduodenectomy around the world. HPB (Oxford) 2015;17:1145-54. [PubMed: 26373586]

7. Molinari E, Bassi C, Salvia R, et al. Amylase value in drains after pancreatic resection as predictive factor of postoperative pancreatic fistula: results of a prospective study in 137 patients. Ann Surg 2007; 246:281-287 [PubMed: 17667507]

8. Giglio M, Spalding A, Giakoustidis A, et al. Meta-analysis of drain amylase content on postoperative day 1 as a predictor of pancreatic fistula following pancreatic resection. BJS 2016; 103: 328-336

9. Bassi C, Molinari E, Malleo G, et al. Early versus late drain removal after standard pancreatic resections: results of a prospective randomized trial. Ann Surg 2010; 252(2): 207-214 [PubMed: 20622661]

10. Strasberg SM, Linehan DC, Hawkins WG. The accordion severity grading system of surgical complications. Ann Surg 2009; 250(2): 177-186. [PubMed: 19638919]

11. Bassi C, Dervenis C, Butturini G, et al. Postoperative pancreatic fistula: an international study group (ISGPF) definition. Surgery 2005; 138(1): 8-13. [PubMed: 16003309]

12. Wente MN, Bassi C, Dervenis C, et al. Delayed gastric emptying (DGE) after pancreatic surgery: a suggested definition by the International Study Group of Pancreatic Surgery (ISGPS). Surgery 2007; 142: 761-768. [PubMed: 17981197]

13. Moskovic DJ, Hodges SE, Wu MF, et al. Drain data to predict clinically relevant pancreatic fistula. HPB 2010; 12(7):472-81. [PubMed: 20815856]

14. Kawai M, Tani M, Terasawa H, et al. Early removal of prophylactic drains reduces the risk of intraabdominal infections in patients with pancreatic head resection: prospective study for 104 consecutive patients. Ann Surg 2006; 244(1): 1-7. [PubMed: 16794381]

15. Adachi T, Kuroki T, Kitasato A, et al. Safety and efficacy of early drain removal and triple-drug therapy to prevent pancreatic fistula after distal pancreatectomy. Pancreatology 2015; 15(4): 411416. [PubMed: 26073457]

16. McMillan M, Malleo G, Bassi C, et al. Multicenter, prospective trial of selective drain management for pancreatoduodenectomy using risk stratification. Ann Surg 2017;265(6):1209-1218. [PubMed: 27280502]

17. Callery MP, Pratt WB, Kent TS, et al. A prospectively validated clinical risk score accurately depicts pancreatic fistula after pancreatoduodenectomy. J Am Coll Surg 2013; 216(1): 1-14. [PubMed: 23122535] 
18. Beane JD, House MG, Ceppa EP, et al. Variation in Drain Management After

Pancreatoduodenectomy: Early Versus Delayed Removal. Ann Surg 2017 Doi: 10.1097/SLA. 0000000000002570. [Epub ahead of print] 


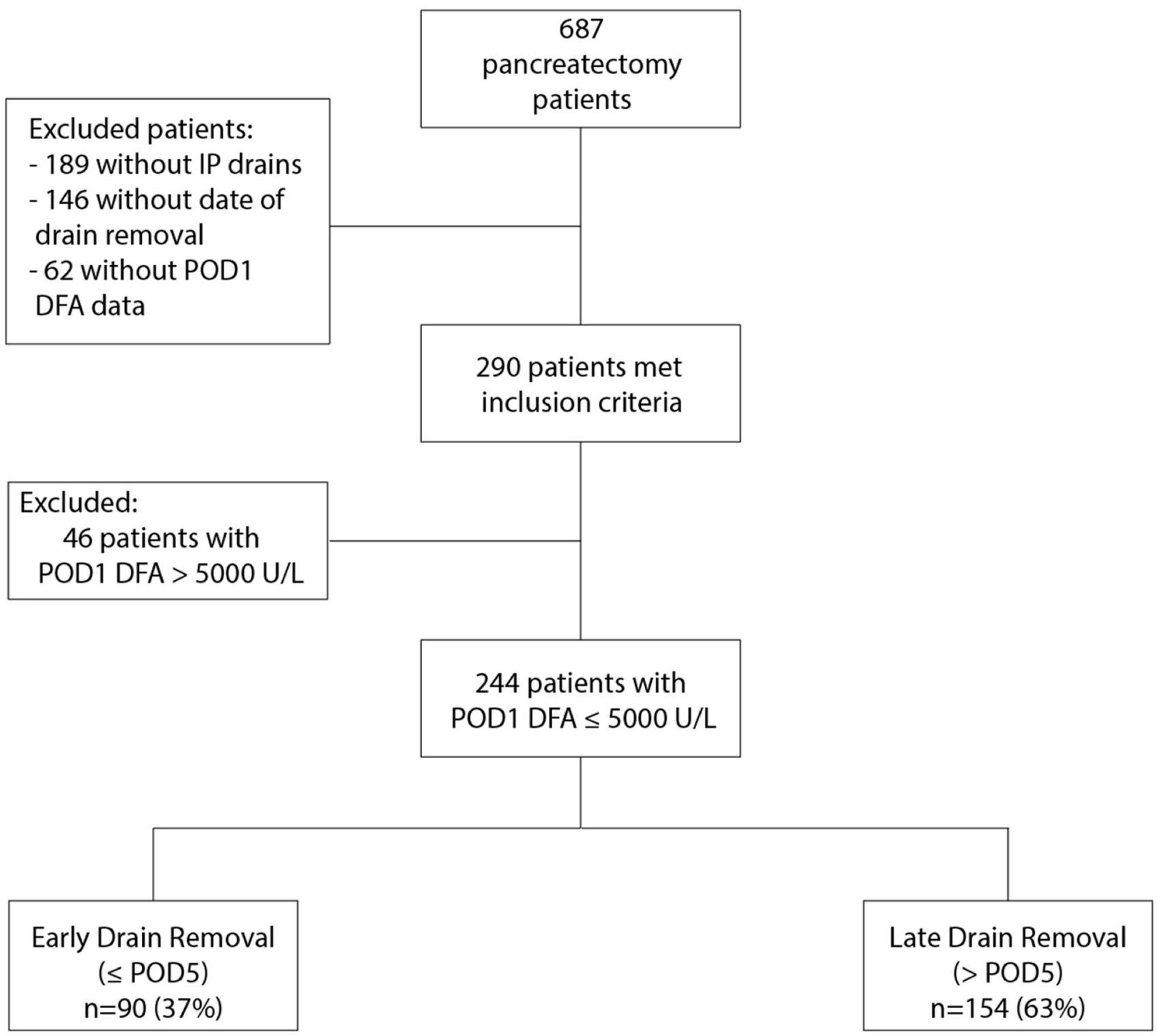

Figure 1.

Patient Selection Strategy

IP: Intraperitoneal

POD: Postoperative day

DFA: Drain fluid amylase 

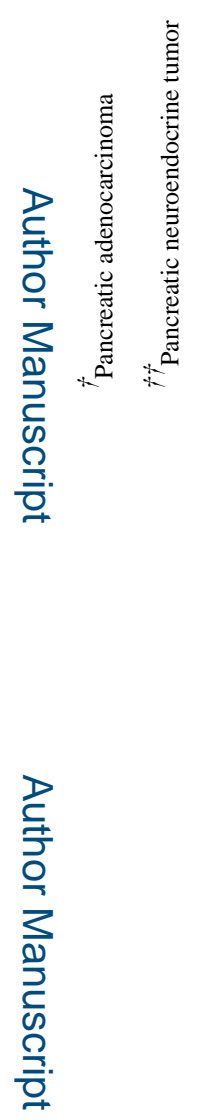

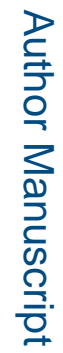

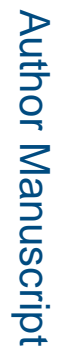

J Surg Res. Author manuscript; available in PMC 2020 April 01. 


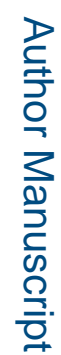

론

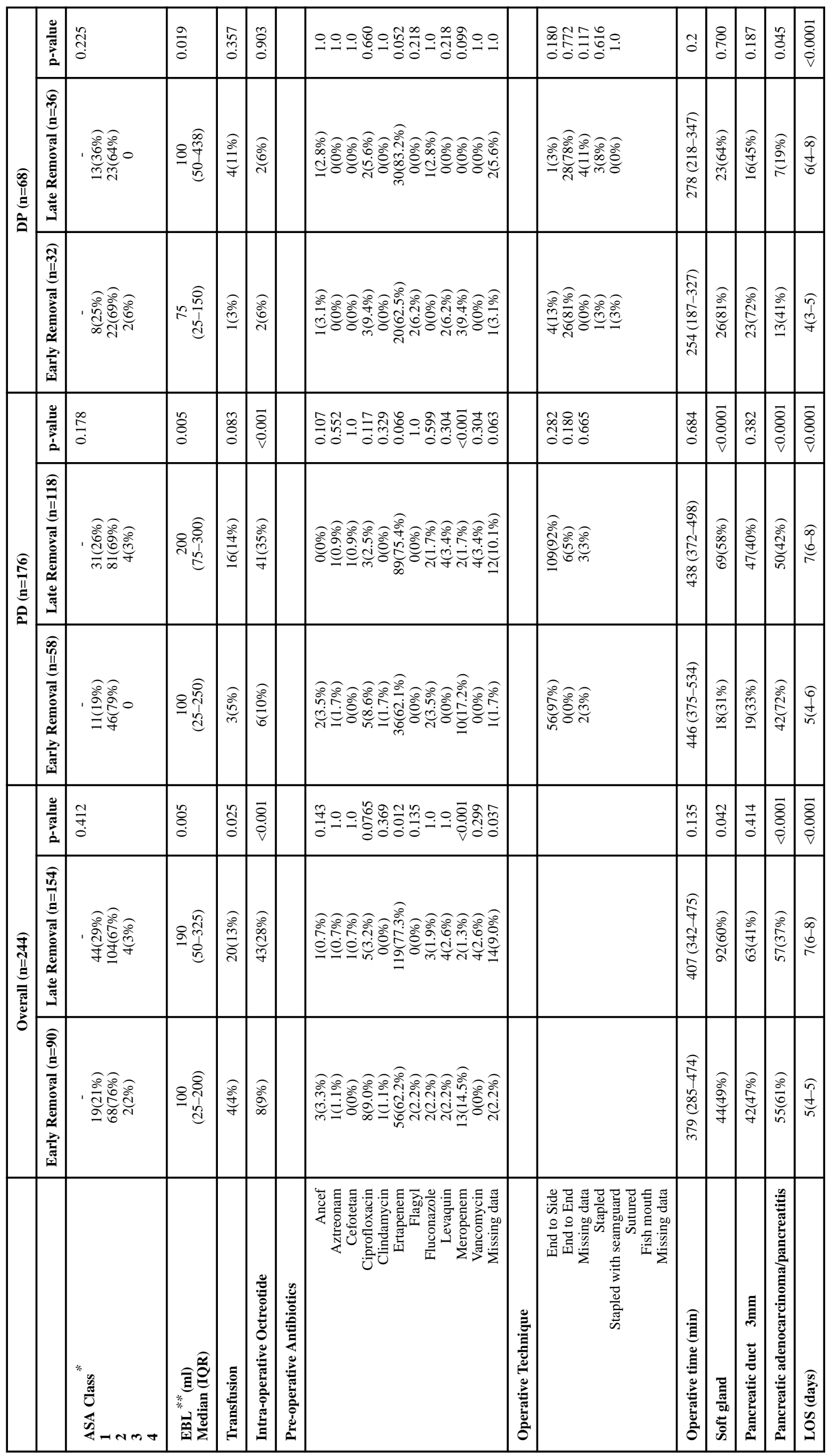




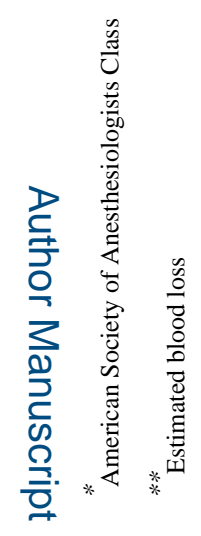

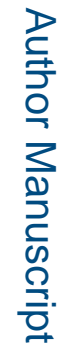

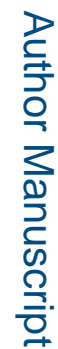

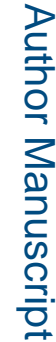




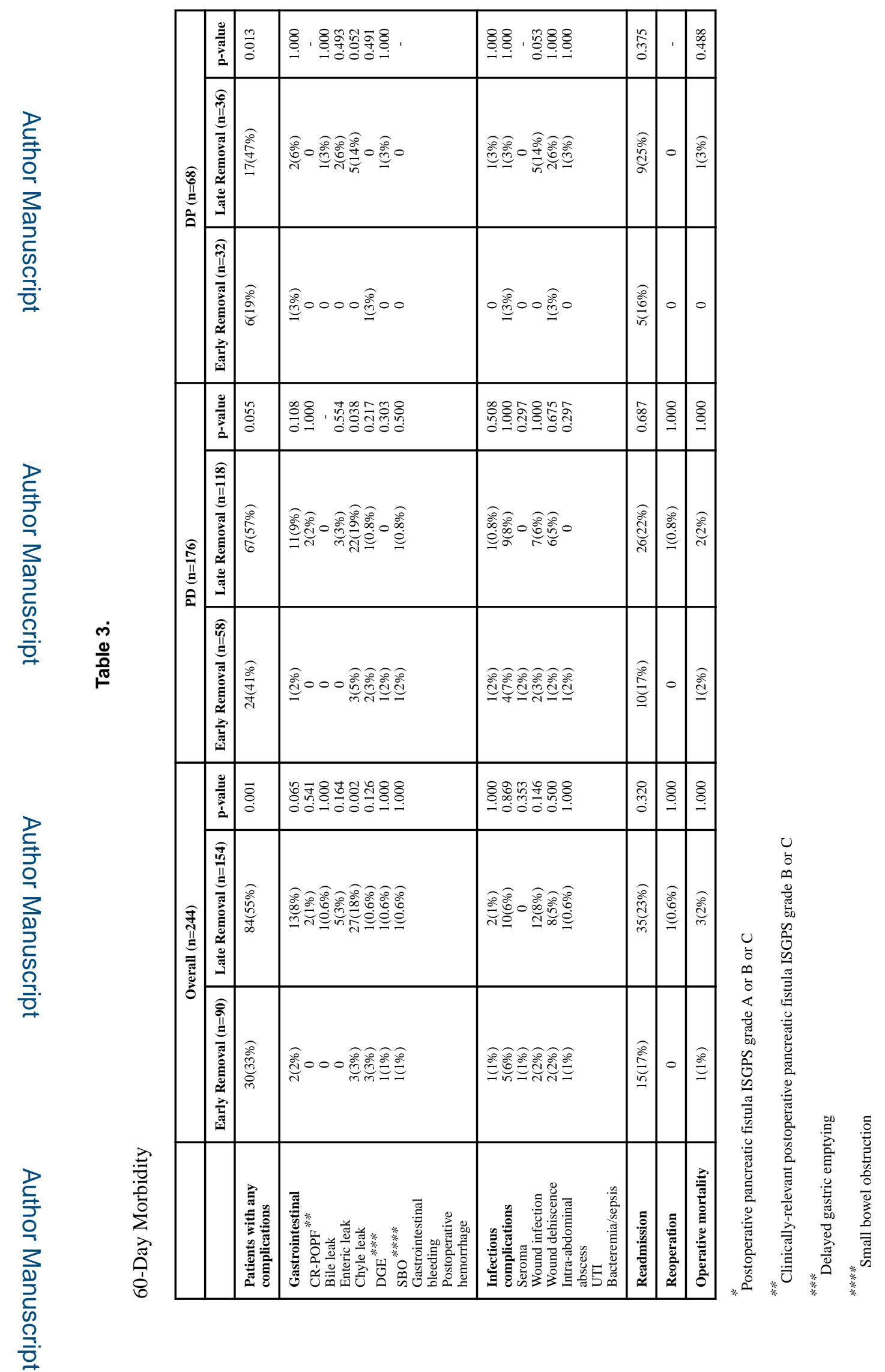

J Surg Res. Author manuscript; available in PMC 2020 April 01. 